\title{
Investigation of Phosphodiesterase 5A (PDE5A) Inhibitors by Pharmacophore Modeling, Virtual Screening and Molecular Docking Approach
}

\author{
Manish Sudesh Bhatia ${ }^{1}$, Amol Shantinath Sherikar ${ }^{2 *}$ \\ ${ }^{1}$ Department of Pharmaceutical Chemistry, Bharati Vidyapeeth College of Pharmacy, Kolhapur, Near Chitranagri, Kolhapur-416 013 (MS) India. \\ ${ }^{2}$ Department of Pharmaceutical Chemistry, Tatyasaheb Kore College of Pharmacy, Warananagar, Tal-Panhala, Dist-Kolhapur-416 113 (MS) India.
}

\begin{tabular}{|c|c|}
\hline ARTICLE INFO & ABSTRACT \\
\hline Article history: & \multirow{10}{*}{$\begin{array}{l}\text { A taxon of accelerator phosphodiesterases (PDEs) corresponds from phosphodiesterase- } 1 \text { to phosphodiesterase- } \\
12 \text { being presently acquainted that inactivates cAMP and cGMP. Owing to this there's no sGC mediate } \\
\text { activation of cGMP/cAMP that regulates vasorelaxation. This project was undertaken to grasp perceptions into } \\
\text { molecular mechanisms and structural wants that area unit crucial for potential inhibition of PDE5A. During } \\
\text { this analysis PDE5A supermolecule was elite and pharmacophore model was generated, virtual screening was } \\
\text { completed to urge hit compounds against reference shared feature pharmacophore, the hit compounds were } \\
\text { docked with PDE5A proteins. Hydrogen bond acceptor, Hydrogen bond donor and aromatic } \\
\text { rings/hydrophobicity are the major phamacophoric features displayed by developed pharmacophore model. The } \\
\text { hit compounds were obtained by virtual screening; compounds were further sorted for Lipinski rule of five } \\
\text { before docking. Compounds that fulfill all properties of Lipinski rule of five were docked with proteins. They fit } \\
\text { appropriately in the pocket of proteins which demonstrated the soundness and stability of ligand compounds. It } \\
\text { is suggested that these compounds can be used in the treatment of diseases and disorders of vasculatures. }\end{array}$} \\
\hline Received on: 08/07/2017 & \\
\hline Accepted on: 20/08/2017 & \\
\hline Available online: $30 / 09 / 2017$ & \\
\hline Key words: & \\
\hline Phosphodiesterases, PDE5A, & \\
\hline Vasorelaxation, & \\
\hline Pharmacophor and Virtual & \\
\hline & \\
\hline & \\
\hline
\end{tabular}

\section{INTRODUCTION}

Phosphodiesterase from vascular smooth muscle catalyzes hydrolyzation of cGMP to inactive product. There are 12 isozymes of phosphodiesterases have been acknowledged in mammalian tissues. Out of 12 isozymes of phosphodiesterase the regulation of cGMP concentration in vascular smooth muscle is associated with phosphodiesterase-5 (PDE5) (Lincoln et al., 1989; Beavo et al., 1995). Sildenafil and tadalafil are inhibitor of PDE5 that has been used to treat erectile dysfunction in humans (Boolell et al., 1996; Boolell et al., 1996; Goldstein et al., 1998). NO synthases produces nitric oxide (NO) constitutively in the lung from vascular endothelium and the airway epithelia (Bohle et al., 2000; German et al., 2002). Depending on alveolar ventilation local NO production regulates pulmonary perfusion to guarantee optimized ventilation distribution (Ide et al., 1999;

* Corresponding Author

Email: amol.sherikar@gmail.com
Grimminger et al., 1995; Sherikar et al., 2015). The regulation of nitric oxide synthase activity is based on transcriptional and posttranslational redox-based modulation level (Michelakis et al., 2003). NO, prostaglandins and natriuretic peptides activates common signalling pathway of endogenous vasodilators like cAMP and cGMP. Phosphodiesterases with different tissue sharing and substrate specificity inactivate cAMP and cGMP (Ahn et al., 1991; Schermuly et al., 2001). The PDE inhibitors control the level of cAMP and cGMP differently depending on their selectivity profile. These secondary messengers are stable which boost the activity. Therefore, they might offers as remedial mechanism to boost and lengthen prostanoid and NO related vascular effects.

The applicability and effectiveness of this approach has been verified in numerous experiments (Weimann et al., 2000; Tanabe et al., 1990). PDE5A was the foremost cGMP-selective PDE to be discovered and is also activated by cGMP. At the present time pharmacophore design approach is a pioneer tool in discovery of drug molecule. 
Many pharmacophore designing and modelling approaches were successfully and extensively utilized in virtual screening, de novo designing and lead improvement. Many ligand and structure based strategies are also developed (Yang et al., 2010; Tripuraneni et al., 2016). Pharmacophores are used as queries for getting better likely lead molecules from structural databases. The obtained lead is used for designing molecules with precise structural attributes and for evaluating resemblance and diversity of molecules (Radwan et al., 2015; Çifci G et al., 2012). Similarly, Virtual screening is a computational process used in the areas of drug discovery and development to explore libraries of small molecules which can be properly bound to their target proteins or enzymes while docking is a phenomenon of predicting the orientations of molecules in the bounded stable complex (Blount et al., 2004; Polymeropoulos et al., 2006; Chandrasekaran et al., 2011; Chien-yu Chen et al., 2009; Kay1k et al., 2017). In this regards, there is necessitate to investigate more molecules specifically targeting PDE/PDE5/PDE5A for its inhibitory potential to obtain a precise structure and phenomenon for vasorelaxation of smooth muscle. Inhibition of PDE5A is the main target for binding of drug molecule to produce smooth muscle relaxation potency.

Herein, we tend to report the application of pharmacophore modeling, virtual screening and molecular docking for PDE5A inhibitors. We undertake above study to recognize intuitions into molecular mechanisms and structural necessities essential for PDE5A inhibition. The novel and potent PDE5A inhibitors can design by using the above information.

\section{MATERIALS AND METHODS}

Methodology used in this work is shown in Figure 1.

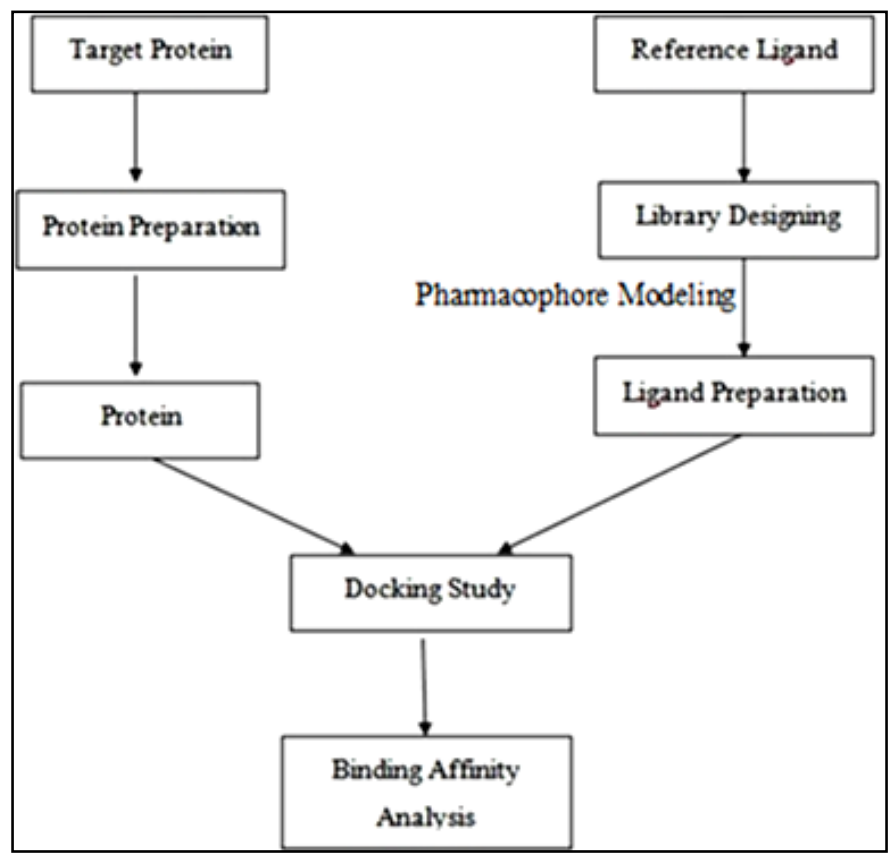

Fig. 1 Docking and binding affinity analysis of the target protein and modified inhibitors.

\section{Selection and Preparation of proteins}

RCSB PDB is principally a database that contains X-ray crystallographic and nuclear magnetic resonant 3D structures of proteins and nucleic acids (Berman et al., 2008). In protein data bank (RCSB PDB) for structural bioinformatics, the experimental method was selected as $\mathrm{X}$-ray crystallography with an $\mathrm{X}$-ray resolution of 1.0-2.0 $\mathrm{A}^{0}$. 1XOZ is a 3D structure of PDE5A protein in complex with 6-benzo[1,3]dioxol-5-yl-2-methyl2,3,6,7,12,12a-hexahydro-pyrazino[1',2':1,6]pyrido[3,4-b]indole-

1,4-dione ligand with a molecular formula $\mathrm{C}_{22} \mathrm{H}_{19} \mathrm{~N}_{3} \mathrm{O}_{4}$. The pharmacophore modeling studies were performed on LigandScout 4.1 Essential (Demo Version, Inte:Ligand GmbH, Vienna, Austria) (Wolber et al., 2005).

\section{Pharmacophore generation}

Pharmacophore hypotheses were generated for reference ligand (Tadalafil) and test ligand using LigandScout software package. Hydrogen bond doner, hydrogen bond acceptor, positive ionizable and aromatic were the common pharmacophoric feature found in every hypothesis. Shared feature pharmacophore was designed which showed the importance of occurrence of common features for inhibition of PDE5A.

\section{Virtual screening of hit compounds against shared feature pharmacophore}

All the molecules were aligned with the shared feature pharmacophore model. Virtual screening was done against shared feature pharmacophore to obtain hit compounds.

\section{Validation of Hit compounds}

After virtual screening the hit compounds were obtained and then validated by screening of Lipinski rule of five. It states that drug-like compound must have hydrogen bond donor (HBD) less than 5, hydrogen bond acceptor (HBA) less than 10, molecular weight not more than $500 \mathrm{Da}$ and $\log \mathrm{P}$ ranges between 0-5 (Pollastri et al.,2010).

\section{Docking of Hit compounds with PDE5A proteins}

The hit compounds were docked in PDB ID: 1XOZ by replacing reference inhibitor ligand. A systematic search was performed to obtain the ligand with lowest energy of interaction. The low energy conformations thus obtained, were optimized till they reached gradient energy of $0.001 \mathrm{kcal} / \mathrm{mol}$. Docking studies were carried out on Vlife molecular docking suite 3.5 by using Biopredicta.

\section{RESULT AND DISCUSSION}

$1 \mathrm{XOZ}$ protein ligand binding domain consists of total 364 amino acid residue and composed of A chain. Pharmacophore investigation is measured as an essential segment of drug design. Hydrogen bond acceptor (HBA), hydrogen bond donor (HBD) and aromatic rings (AR) are the major feature of pharmacophore model generated by LigandScout for the selected protein data set of 
vascular smooth muscle relaxation. In each pharmacophore model of selected proteins the red arrows represent hydrogen bond acceptor, green arrow represents hydrogen bond donor and yellow spheres represent an aromatic ring. Numerous excluded volumes were also produced in the models to demonstrate the space balancing. The representative pharmacophores of PDE5A protein ligands are shown in Figure 2. Pharmacophore models of selected protein data sets were aligned together on the basis of ligand structure to generate a shared feature pharmacophore and interacting amino acid residues shown in Figure 3.
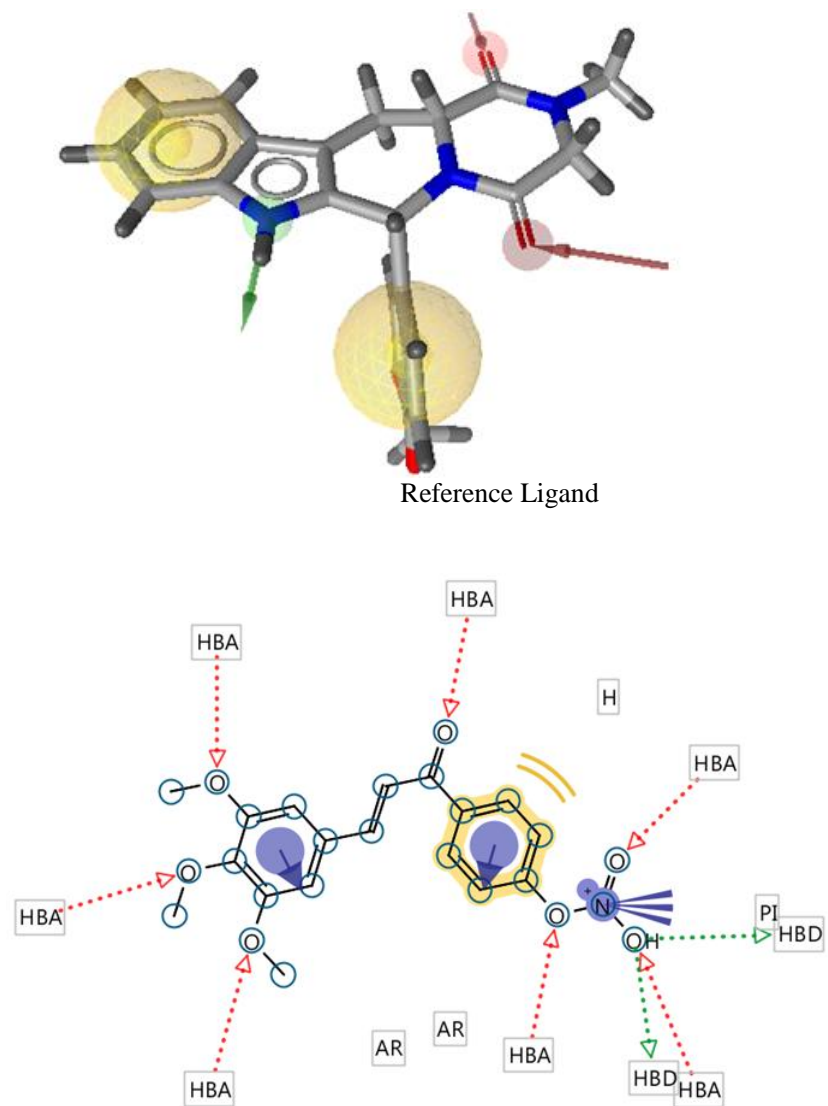

AI6

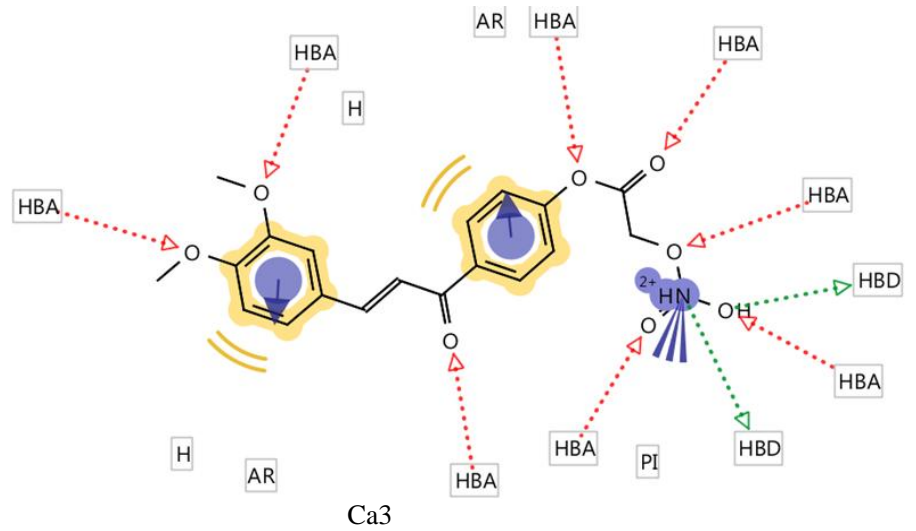

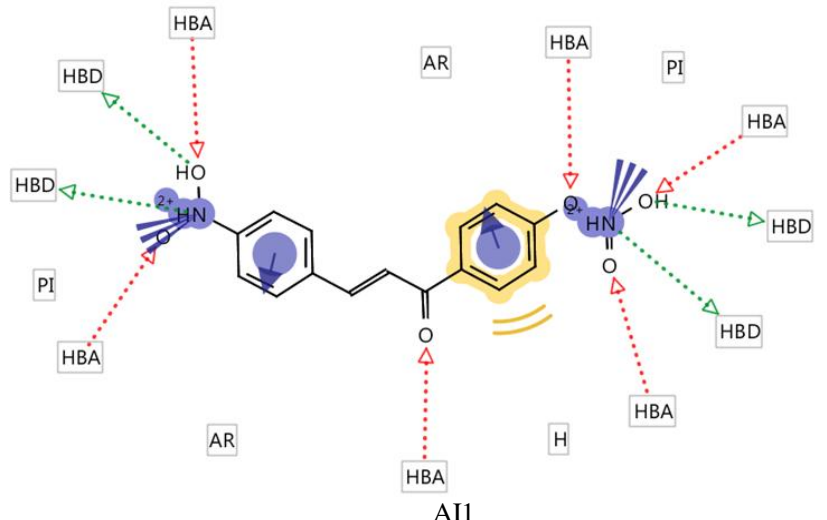
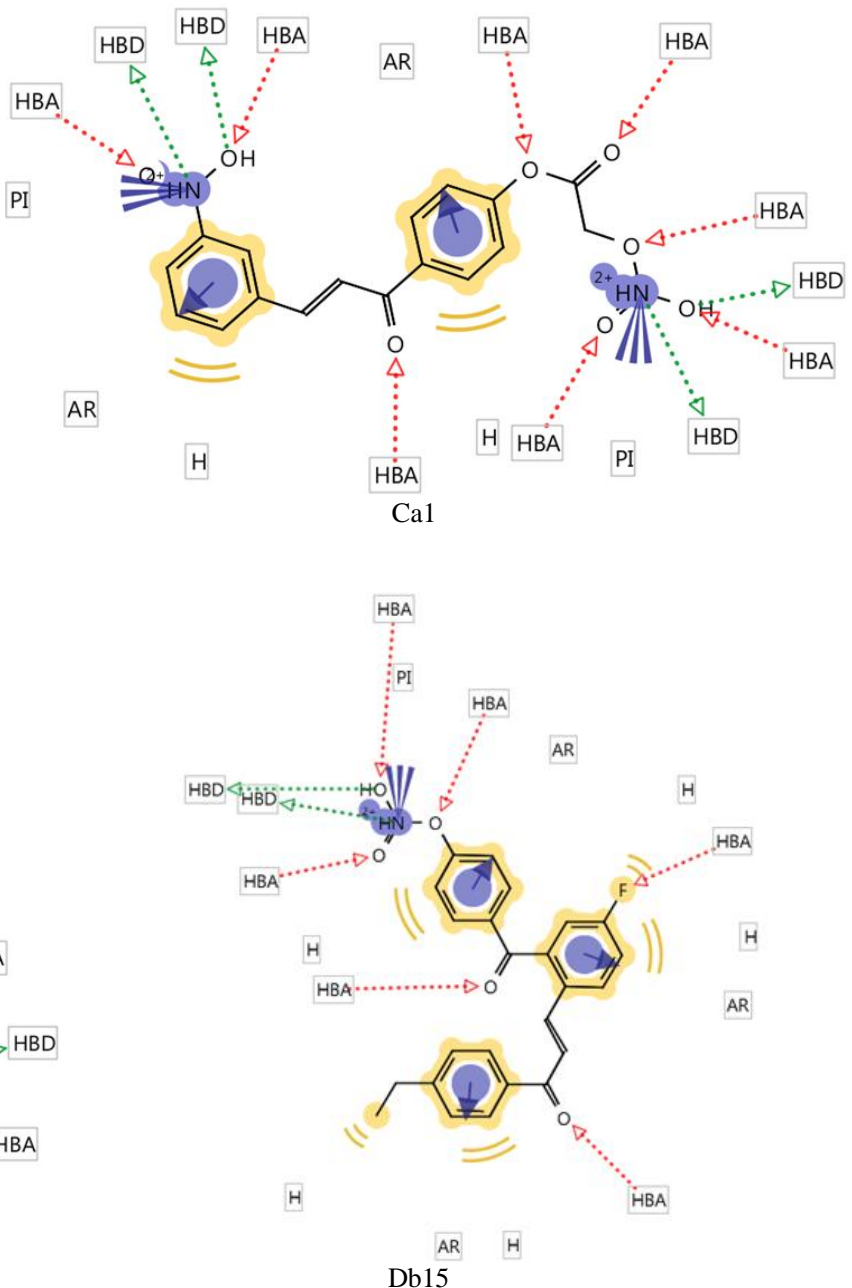

Fig. 2: Pharmacophore model of $1 \mathrm{XOZ}$ protein with reference ligand, AI1, AI6, Ca1, Ca3 and Db15. 


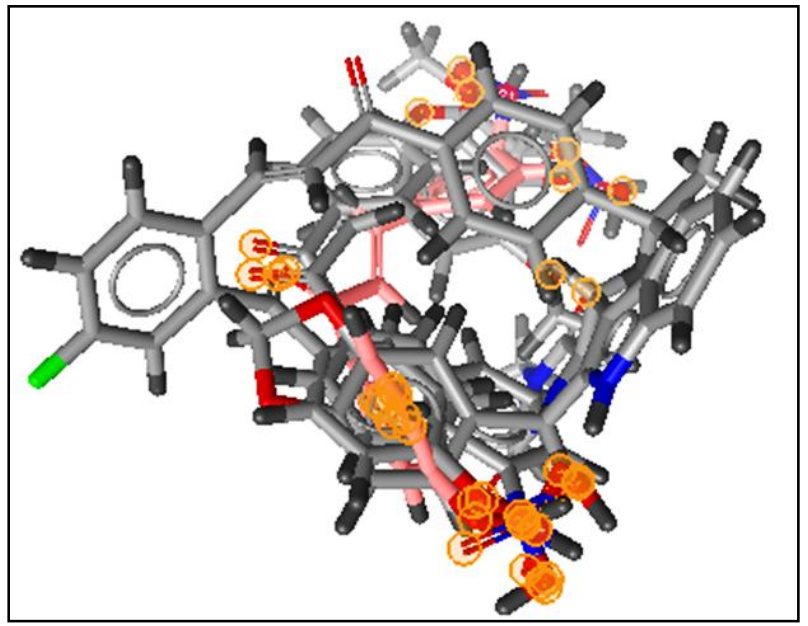

Alignment of reference and ligands with shared feature pharmacophores

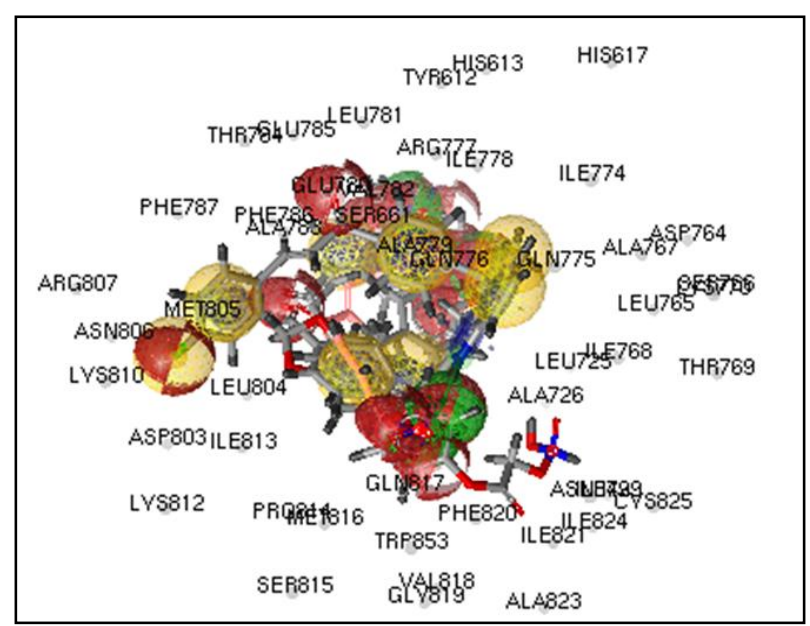

Shared feature pharmacophores and interacting amino acid residues

Fig. 3: Model of shared feature pharmacophore of $1 \mathrm{XOZ}$ protein structures ligands.

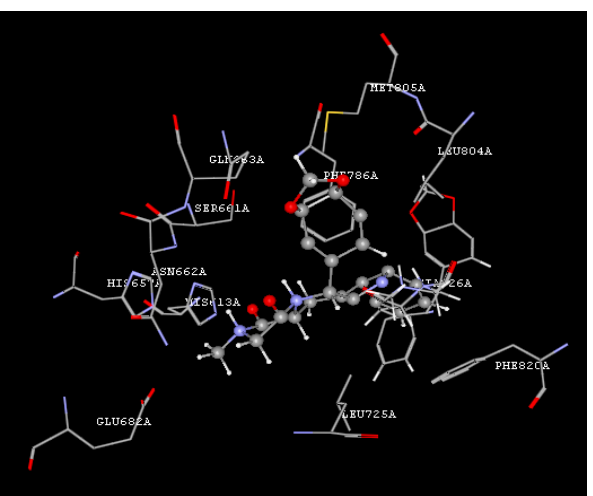

Reference Tadalafil

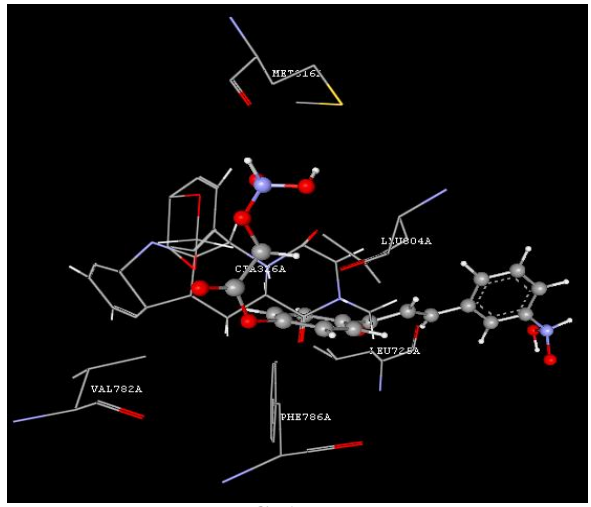

Ca1

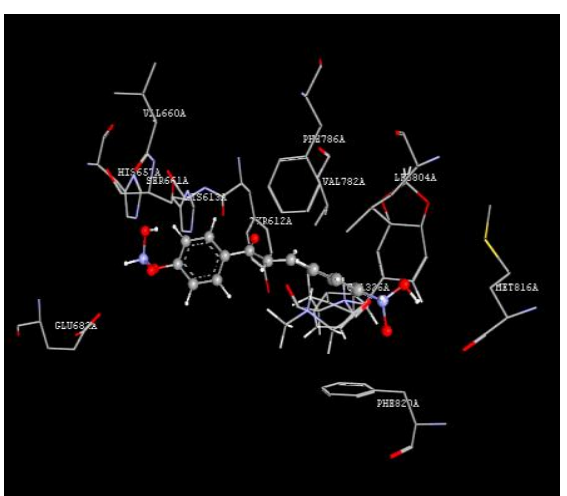

AI1

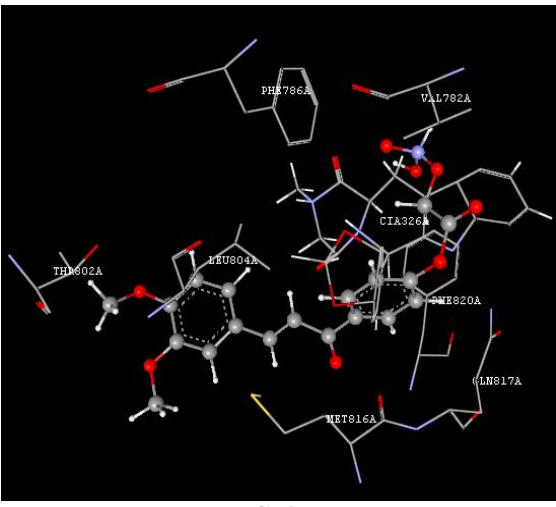

$\mathrm{Ca3}$

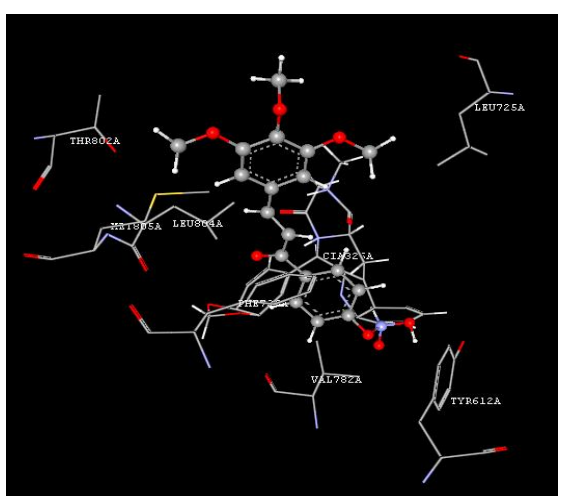

AI6

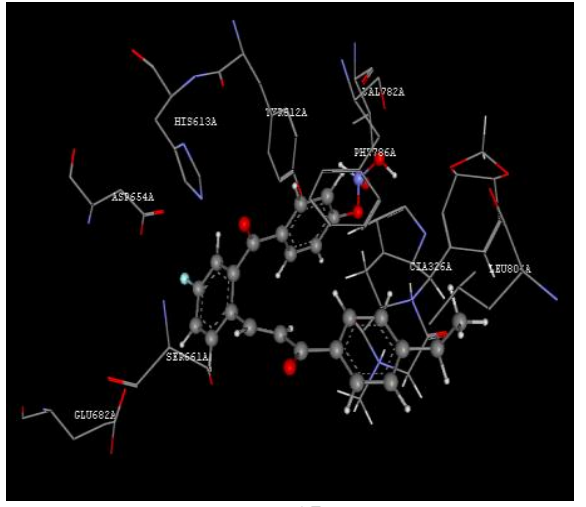

Db15

Fig. 4 Docking results of Reference (Tadalafil), AI1, AI6, Ca1, Ca3 and Db15 with 1XOZ protein.

In the Virtual screening hit compounds analogous to share feature pharmacophore model were obtained. The hit compounds were then checked for Lipinski rule of five, only five compounds were fulfilling all the rules of Lipinski, i.e., molecular weight $<500 \mathrm{Da}, \mathrm{HBD}<5, \mathrm{HBA}<10$ and $\log \mathrm{P}$ between $0-5$. The hit compounds which fulfilled Lipinski rule of five are shown in Table 1.

The docking study is implemented to calculate and predict the different interaction between drug and receptor. All satisfied compounds were docked with PDE 5A protein; in every docked complex the common interacting amino acid residues were same as that of pharmacophore model. The five compounds which fulfilled Lipinski rule of five and their best docking results are shown in Table 2. The best arrival poses with receptor interaction of these molecules are shown in figure 4.

All these five compounds are best suitable to use as drugs as they fulfill all the properties of Lipinski's rule so they will demonstrate fewer side effects as compared to the drugs available in the market. It is suggested that these five compounds can be used in the treatment of vasculature diseases and disorders. 
Table 1: Chemical structures, molecular formulae, molecular weights, $\log$, HBD and HBA of reference and hits fulfilling the Lipinski rule of five.

\begin{tabular}{|c|c|c|c|c|c|c|}
\hline & Compound Structure & $\begin{array}{c}\text { Molecular } \\
\text { formula }\end{array}$ & Molecular Weight & $\log P$ & HBD & HBA \\
\hline Reference Ligand & & $\mathrm{C}_{21} \mathrm{H}_{17} \mathrm{~N}_{3} \mathrm{O}_{4}$ & 375.37738 & 389.411 & 01 & 02 \\
\hline AI1 & & $\mathrm{C}_{15} \mathrm{H}_{10} \mathrm{~N}_{2} \mathrm{O}_{6}$ & 314.2497 & 3.426 & 04 & 06 \\
\hline AI6 & & $\mathrm{C}_{18} \mathrm{H}_{17} \mathrm{NO}_{7}$ & 359.33008 & 3.824 & 02 & 07 \\
\hline $\mathrm{Ca} 1$ & & $\mathrm{C}_{17} \mathrm{H}_{12} \mathrm{~N}_{2} \mathrm{O}_{8}$ & 372.28578 & 3.452 & 04 & 08 \\
\hline $\mathrm{Ca} 3$ & & $\mathrm{C}_{19} \mathrm{H}_{17} \mathrm{NO}_{8}$ & 387.34018 & 3.250 & 02 & 08 \\
\hline Db15 & ? & $\mathrm{C}_{24} \mathrm{H}_{18} \mathrm{FNO}_{5}$ & 419.4018232 & 3.542 & 02 & 06 \\
\hline
\end{tabular}

Table 2: Comparison of bonding types of compounds which demonstrated ideal docking results along with binding energy.

Compound Code
Reference Ligand
AI1
AI6
Ca1
Ca3
Db15

Hydrophobic and VDW Interactions
Hydrogen bond and VDW Interactions
VdW and hydrophobic interaction
VdW, Hydrogen bonding and hydrophobic interaction
VdW, Hydrogen bonding and hydrophobic interaction
VdW, Hydrogen bonding and hydrophobic interaction

Binding Energy

$-49.978155$

$-43.784469$

$-28.377318$

$-43.28555$

$-41.577673$

$-40.925281$ 


\section{CONCLUSION}

The pharmacophore model is a very promising tool for new lead compounds discovery and development. Two common approaches used in pharmacophore modelling are ligand based and structured based. Ligand-based pharmacophore modelling adapts the superposition of potent compounds and isolating shared structural features necessary for the biological activity of molecule whiles structure based pharmacophore modelling adapts the mechanism of examining promising interactions between receptor and ligand (Yang et al., 2010). The ligand based pharmacophore modelling approach is utilized in this research work. Combinations of the pharmacophore modelling, virtual screening and molecular docking positively give possible inhibitors that can have endless influence for various experimental studies in vasculature diseases.

\section{ACKNOWLEDGEMENT}

Authors are grateful to Principal Bharati Vidyapeeth College of Pharmacy, Kolhapur for availing all necessary amenities to do above research. We are also thankful to Principal and Management of Tatyasaheb Kore College of Pharmacy, Warananagar for motivation and support.

Financial support and sponsorship: This work was supported financial assistance from AICTE, New Delhi as a Research Promotion Scheme (RPS) (file no. 8023/RID/RPS/20//2011-12).

Conflict of Interests: There are no conflicts of interest.

\section{REFERENCES}

Ahn HS, Foster M, Cable M, Pitts BJ, Sybertz EJ. Ca/CaM stimulated and cGMP-specific phosphodiesterases in vascular and nonvascular tissues. Adv Exp Med Biol, 1991; 308:191-197.

Beavo JA. Cyclic nucleotide phosphodiesterases: functional implications of multiple isoforms. Physiol. Rev, 1995; 75:725-748.

Berman HM. The protein data bank: A historical perspective. Acta Crystallographica Section A Foundation of Crystallography, 2008; 64:88-95.

Blount MA, Beasley A, Zoraghi RR, Sekhar KR, Bessay EP, Francis SH, Corbin JD. Binding of Tritiated Sildenafil, Tadalafil, or Vardenafil to the Phosphodiesterase-5 Catalytic Site Displays Potency, Specificity, Heterogeneity and cGMP Stimulation. Molecular Pharmacology, 2004; 66:144-152.

Boolell M, Allen MJ, Ballard SA. Sildenafil: an orally active type 5 cyclic GMP-specific phosphodiesterase inhibitor for the treatment of penile erectile dysfunction. Int J Impot Res, 1996; 8:47-52.

Boolell M, Gepi-Attee S, Gingell JC, Allen MJ. Sildenafil a novel effective oral therapy for male erectile dysfunction. Br J Urol, 1996; 78:257-261.

Bohl RM, Hartmann E, Kinfe T, Ermert L, Seeger W, Fink L. Cell type-specific mRNA quantitation in nonneoplastic tissues after laser assisted cell picking. Pathology, 2000; 68;191-195.

Chandrasekaran M, Sakkiah S, Lee KW. Combined ligand based pharmacophore modeling, virtual screening methods to identify critical chemical features of novel potential inhibitors for phosphodiesterase-5. Journal of the Taiwan Institute of Chemical Engineers, 2011; 42:709-718.

Chen C, CHANG Y, BAU D, HUANG H, Tsai F, TSA C, Yuchian Chen C. Discovery of potent inhibitors for phosphodiesterase 5 by virtual screening and pharmacophore analysis. Acta Pharmacologica Sinica, 2009; 30:1186-1194.
Cifci G, Aviynte V, Akten ED. Molecular Docking Study Based on Pharmacophore Modiling for Novel Phosphodiesterase IV inhibitors. Molecular Informatics, 2012; 31:459-471.

German Z, Chambliss KL, Pace MC, Amet UA, Lowenstein CJ, Shaul PW. Molecular basis of cellspecific endothelial nitric-oxide synthase expression in airway epithelium. J Biol Chem, 2002; 275:81838189.

Goldstein I, Lue TF, Padma-Nathan H, Roshan RC, Steers WD, Wicker PA. Oral sildenafil in the treatment of erectile dysfunction. N Engl J Med, 1998; 338:397-404.

Grimminger F, Spriestersbach R, Wiessmann N, Walmrath D, Seeger W. Nitric oxide generation and hypoxic vasoconstriction in bufferperfused rabbit lungs. J Appl Physiol, 1995; 78:1509-1515.

Ide H, Nakano H, Ogasa T, Osanai S, Kikuchi K, Iwamoto J. Regulation of pulmonary circulation by alveolar oxygen tension via airway nitric oxide. J Appl Physiol, 1999; 87:1629-1636.

Kayık G, Tüzün NŞ, Durdagi S. Investigation of PDE5/PDE6 and PDE5/PDE11 Selective Potent Tadalafil-like PDE5 Inhibitors Using Combination of Molecular Modeling Approaches, Molecular FingerprintBased Virtual Screening Protocols and Structure-based Pharmacophore Development. Journal of Enzyme Inhibition and Medicinal Chemistry, 2017; 32:311-320

Lincolin TM. Cyclic GMP and mechanism of vasodilation. Pharmacol Ther, 1989; 41:479-502.

Michelakis ED. The role of the NO axis and its therapeutic implication in pulmonary arterial hypertension. Heart Fail Rev, 2003; 8:521.

Pollastri MP. Overview of the rule of five. Current Protocol in Pharmacology. John Wiley \& Sons Inc. 2010.

Polymeropoulos EE, Hogfen R. A Pharmacophore Model for PDE IV Inhibitors. Quantitative Structure Actovity Relationships, 2006; 16:231-234.

Radwan AA. Pharmacophore elucidation and molecular docking studies on phosphodiesterase-5 inhibitors. Bioinformation, 2015; 11:63-66.

Schermuly TR, Krupnik E, Tenor H. Coaerosolization of phosphodiesterese inhibitors markedly enhances the pulmonary vasodilatory response to inhaled iloprost in experimental pulmonary hypertension. Maintenance of lung selectivity. Am J Respir Crit Care Med, 2001; 164:1694-1700.

Sherikar AS, Bhatia MS, Dhavale RP, Bhatia NM, Choudhari PB. Nitric Oxide (NO) and Hydrogen Sulfide (H2S): Molecular Targets for Vascular Muscle Relaxation. Current Trends in Biotechnology and Pharmacy, 2015; 9:412-420.

Tanabe T, Beam KG, Adams BA. Regions of the skeletal muscle dihydropyridine receptor critical for excitation-contraction coupling. Nature, 1990; 346:567-569.

Tripuraneni NS, Azam MA. A combination of pharmacophore modeling, atom-based 3D-QSAR, molecular docking and molecular dynamics simulation studies on PDE4 enzyme inhibitors. Journal of Biomolecular Structure and Dynamics, 2016; 34:2481-2492.

Weimann J, Ullrich R, Hromi J. Sidenafil is a pulmonary vasodilator in awake lambs with acute pulmonary hypertension. Anesthesiology, 2000; 92:1702-1712.

Wolber G, Langer T. LigandScout: 3-D Pharmacophorees Derived from Protein-Bound Ligands and Their Use as Virtual Screening Filters. J. Chem. Inf. Model, 2005; 45:160-169.

Yang YS. Pharmacophore modeling and applications in drug discovery: challenges and recent advances. Drug Discov Today, 2010; 15:440-450.

\section{How to cite this article:}

Bhatia MS, Sherikar AS. Investigation of Phosphodiesterase 5A (PDE5A) Inhibitors by Pharmacophore Modeling, Virtual Screening and Molecular Docking Approach. J App Pharm Sci, 2017; 7 (09): 038-043. 